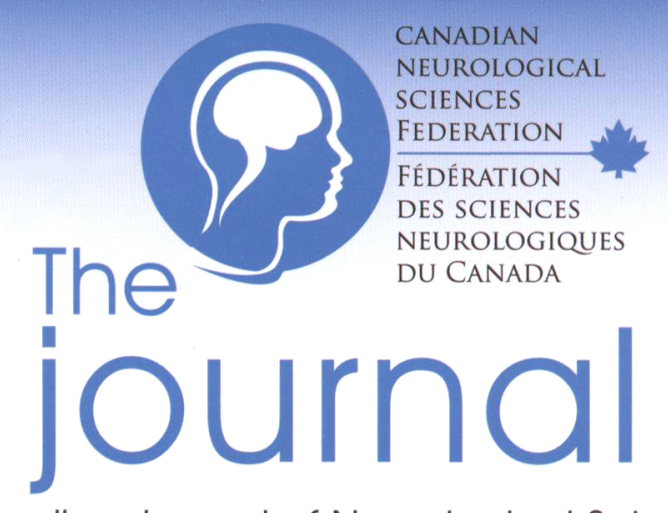

Canadian Journal of Neurological Sciences

Volume 37 Number 3 May 2010
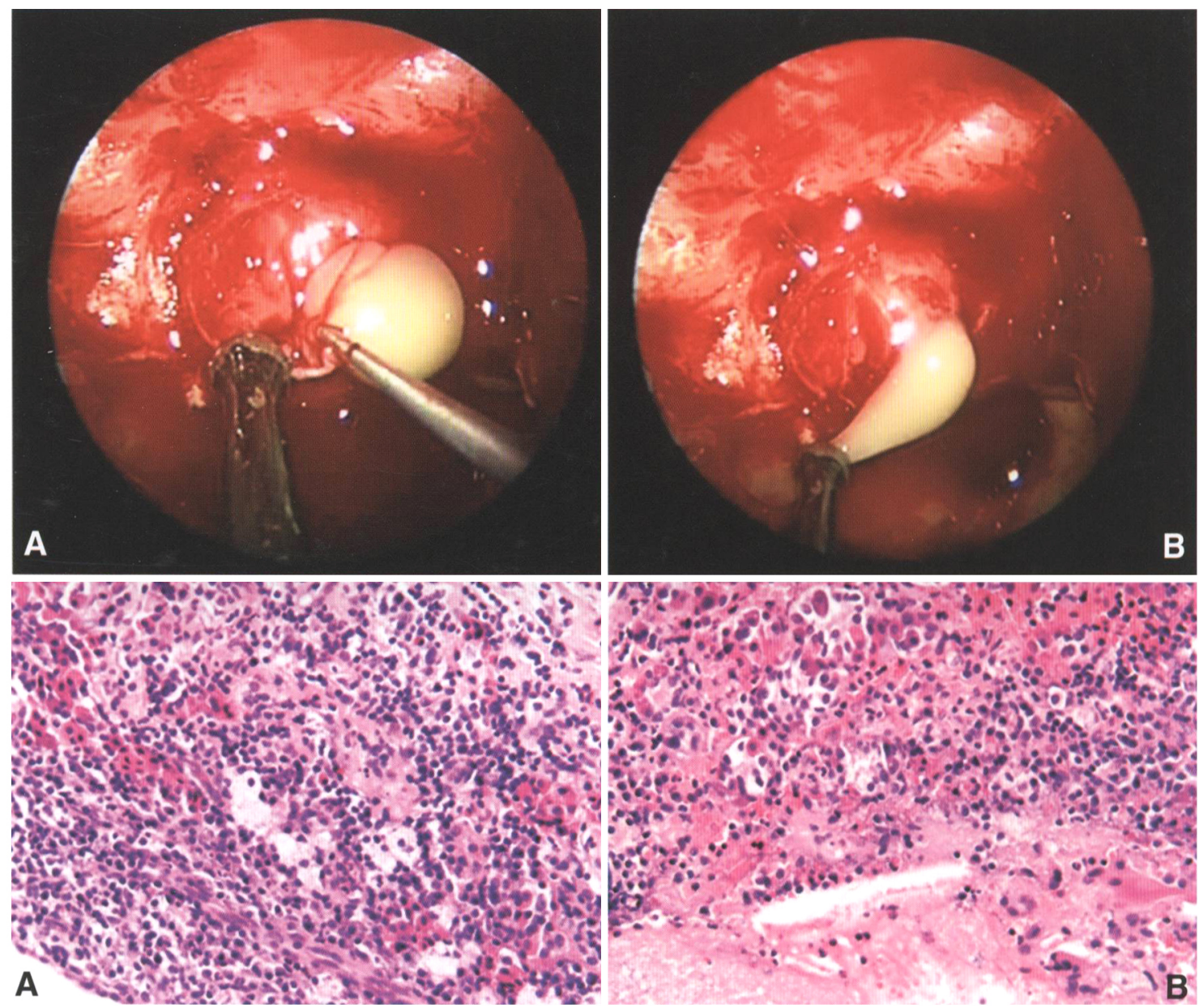

See Page A-2 for figure legend 


\section{Beyond technology}

\section{there's possibility}

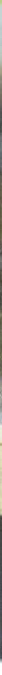

Over 40 years ago Professor Lars Leksell invented Gamma Knife ${ }^{\circledR}$ surgery, a revolutionary, non-invasive way to perform brain surgery that created new treatment possibilities for patients with inoperable brain cancer. Today, Leksell Gamma Knife ${ }^{\circledR}$ Perfexion $^{\mathrm{TM}}$ is the cornerstone of a complete line of stereotactic treatment and radiosurgery solutions that is still creating new possibilities for the most challenging cases of the brain, head \& neck, and body. Find out more at elekta.com/proof.
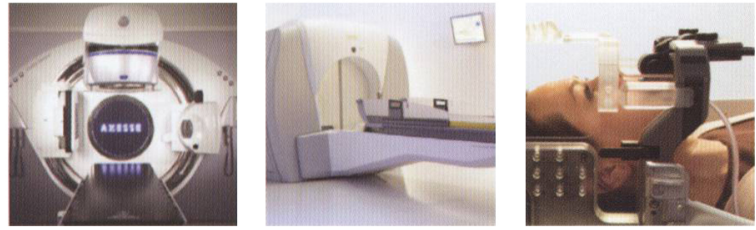

Elekta Axesse $^{\text {TM }}$

Leksell Gamma Knife ${ }^{*}$ Perfexion $^{\mathrm{TM}}$

Extend $^{\text {Th }}$ program 


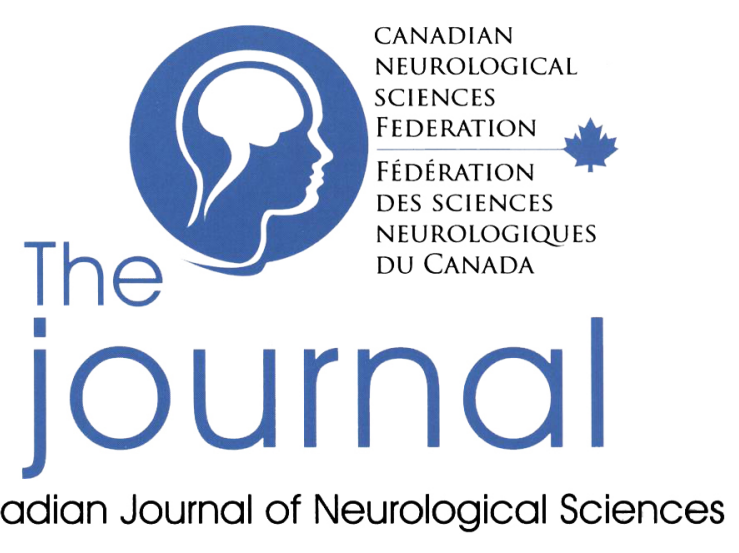

\section{Volume 37 , Number 3 , May 2010}

\section{EDITORIALS}

301 Death and Injury Due to All-Terrain Vehicles - A Disturbing Trend

Patrick McDonald

302 Familial Alzheimer Disease in Canada

Nicolas Dupré, Robert Laforce $\mathrm{Jr}$.

304 What Means Familial?

\section{F. Clarke Fraser}

305 Where are we Going with Drugs to Treat MS? Will Cost Continue to Increase?

Où allons-nous avec les traitements de la SEP? Les coûts vont-ils continuer à augmenter?

Joel Oger, Mona AlKhawajah

309 The Tolerability and Efficacy of Levetiracetam

Jose F. Tellez-Zenteno

310 Canadian Health Care Explained to U.S. Colleagues: the Good, the Bad and the Ugly

J. Max Findlay

\section{HISTORIAL REVIEW}

313 Substantia Nigra and Parkinson's Disease: A Brief History of Their Long and Intimate Relationship

Martin Parent, André Parent

\section{REVIEW ARTICLES}

320 Carotid Revascularization: An Update

Cian J. O'Kelly, Ken S. Butcher, B. Elaine Marchak, J. Max Findlay

336 Autopsy as Gold Standard in FDG-PET Studies in Dementia Pascali Durand-Martel, Dominic Tremblay, Catherine Brodeur, Nancy Paquet

\section{ORIGINAL ARTICLES}

343 Examining Ontario Deaths Due to All-Terrain Vehicles, and Targets for Prevention

Sarah Lord, Charles H. Tator, Sandy Wells
350 Early Onset Epilepsy and Inherited Metabolic Disorders: Diagnosis and Management

Asuri N. Prasad, G.F. Hoffmann

359 A Novel PS1 Gene Mutation in a Large Aboriginal Kindred

Rachel Butler, B. Lynn Beattie, Umamon Puang Thong, Emily Dwosh, Colleen Guimond, Howard H. Feldman, Ging-Yuek Robin Hsiung, Ekaterina Rogaeva, Peter St. George-Hyslop, A. Dessa Sadovnick

365 Deregulated Expression of the Perl and Per2 in Human Gliomas

He-chun Xia, Zhan-feng Niu, Hui Ma, Shuan-zhu Cao, Shao-cai Hao, Zhong-tao Liu, Fan Wang

371 Microembolic Signals in Patients with Systemic Lupus Erythematosus

Mahmoud Reza Azarpazhooh, Naghmeh Mokhber, Elias Orouji, Brian R. Chambers, Mohammad Reza Hatef, Zahra Rezaieyazdi, Sima Sedighi, Mohsen Foroghipoor, Arash Velayati, Morteza Modares Gharavi

376 Levetiracetam in Clinical Practice: Efficacy and Tolerability in Epilepsy

Leonilda Bilo, Maria Fulvia de Leva, Roberta Meo

383 Increasing Use of Disease Modifying Drugs for MS in Canada

Dalia L. Rotstein, Muhammad Mamdani, Paul W. O'Connor

389 Familial Occurrence of Complex Regional Pain Syndrome

Peyman Shirani, Ali Jawaid, Paolo Moretti, Elham Lahijani, Alicia R. Salamone, Paul E. Schulz, Everton A. Edmondson

\section{NEUROIMAGING HIGHLIGHTS}

395 The "Reversal Sign" Following a Massive Left-Hemispheric Stroke

Philippe Huot, François Émond

397 Delayed Neurological Deterioration in ICH Due to Cerebral Infarction

B.K. Menon, J. Singh, M.D. Hill

400 Spinal Cord Transection in a Child After Non-Penetrating Trauma

Fred A. Zeiler, Patrick J. McDonald 


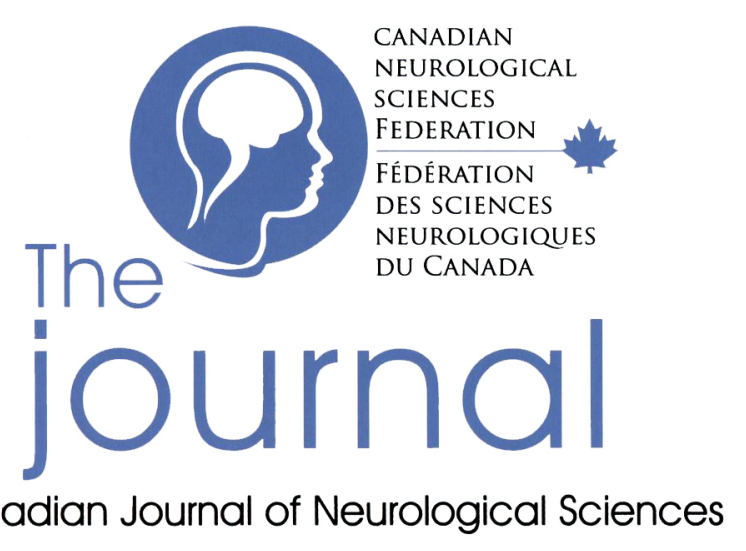

\section{Volume 37 / Number 3 , May 2010}

\section{BRIEF COMMUNICATIONS}

402 Hypophysitis Secondary to a Ruptured Rathke Cleft Cyst Nancy McLaughlin, François Lavigne, Shaun Kilty, France Berthelet, Michel W. Bojanowski

406 Hydroxyzine Hydrochloride in Familial Restless Legs Syndrome

Roberto Di Fabio, Carlo Casali, Rita Vadalà, Francesco Pierelli

408 Middle Cerebral Artery Embolectomy After Failed Mechanical Clot Removal Brian Drake, Gary Redekop

412 Status Epilepticus Due to Hyperfusion Injury Post Cardiac Surgery

Nir Lipsman, Richard Farb, Osman O. Al-Radi, Leanne K. Casaubon

416 Primary Choroid Plexus Papilloma of the Cauda Equina. A Case Report

Ryan DeMarchi, Hisham Al Khalidi, Mahmood Fazl, Juan M. Bilbao

419 Charcot Ankle Arthropathy in CMT1A Exacerbated by Type 2 Diabetes Mellitus

Natalie E. Parks, Timothy J. Benstead

\section{REFLECTIONS}

422 Olive

Mark Bernstein

\section{CORRESPONDENCE}

423 Letters to the Editor

425 Books Received/Books Reviewed

428 Calendar of Events

A-13 Board of Directors/Committee Chairs

A-3 CNSF 2010 Congress Program

IBC CNSF Sponsors

A-9 Information for Authors

A-10 Information for Authors

A-17 Advertisers Index

A-17 Classified Ads

A-22 Annual Congress Registration and Hotel Details 


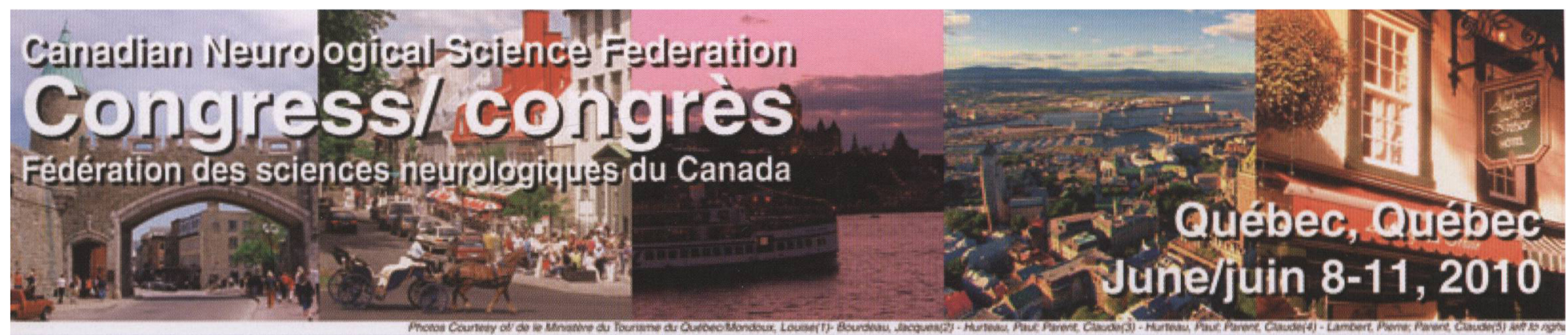

\section{CONGRESS-AT-A-GLANCE}

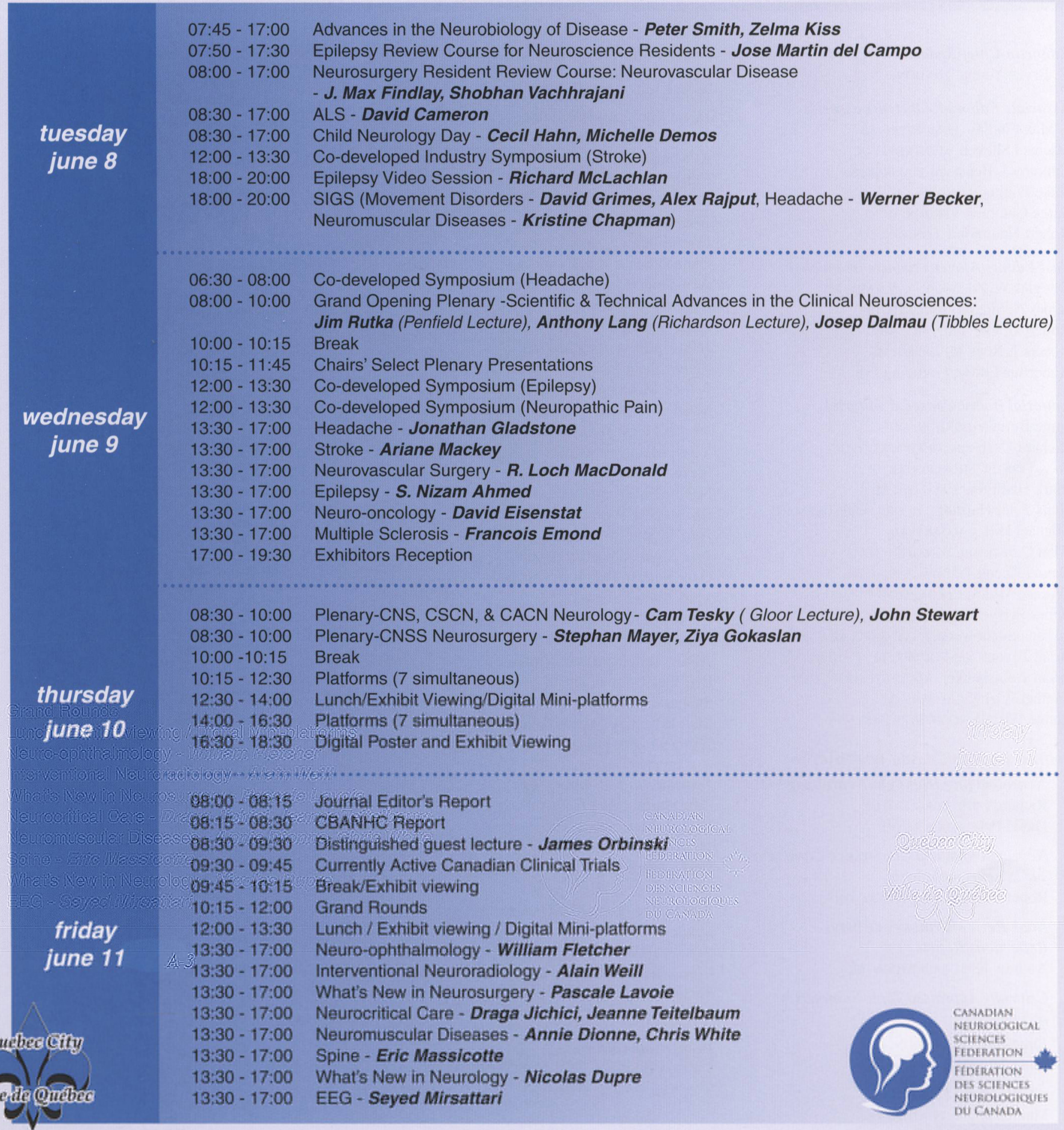




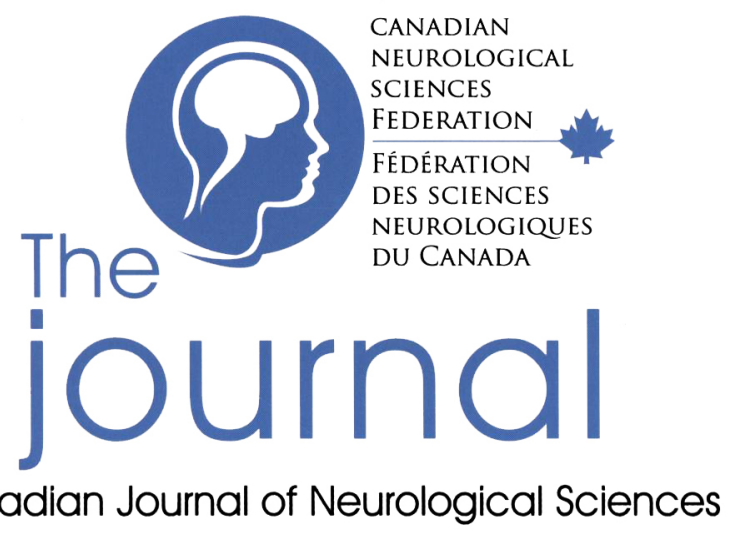

\section{Volume 37 , Number 3 , May 2010}

Editor-in-Chief/Rédacteur en chef

G. Bryan Young LONDON, ON

Associate Editors/Rédacteurs associés

J. Max Findlay EDMONTON, AB

Michael Shevell MONTREAL, QC

Timothy J. Benstead HALIFAX, NS

Mike Poulter LONDON, ON

Serge Gauthier VERDUN, QC

Robert Hammond LONDON, ON

Past Editors/Anciens rédacteurs en chef

Douglas W. Zochodne CALGARY, AB

James A. Sharpe TORONTO, ON

Robert G. Lee CALGARY, AB

Robert T. Ross WINNIPEG, MB

(Emeritus Editor, Founding Editor)

\section{Editorial Board/Conseil d'éditorial}

Jorge Burneo LONDON, ON

Richard Desbiens QUEBEC CITY, QC

David Fortin SHERBROOKE, QC

Mark Hamilton CALGARY, AB

Hans-Peter Hartung DUSSELDORF, GERMANY

Michael Hill CALGARY, AB

Alan C. Jackson WINNIPEG, MB

Daniel Keene OTTAWA, ON

Terence Myles CALGARY, AB

James Perry TORONTO, ON

Oksana Suchowersky CALGARY, AB

Brian Toyota VANCOUVER, BC

Brian Weinshenker ROCHESTER, MN, USA

Samuel Wiebe CALGARY, AB

Elaine Wirrell ROCHESTER, MN, USA

\section{SECTION EDITORS/CONSEIL DE RÉDACTION}

Neuroimaging Highlight/Neuroimagerie

Richard Farb TORONTO, ON

David Pelz LONDON, ON

Neuropathological Conference/Conférence sur la neuropathologie

Robert Hammond LONDON, ON

Book Review/Critiques de livres

Reflections/Reflets

Andrew Kirk SASKATOON, SK

Critically Appraised Topic Summaries

(CATS)

Jorge Burneo LONDON, ON

Mary Jenkins LONDON, ON

e-CPD (Web) Editor

Mandar Jog LONDON, ON
Editorial Review Board/Conseil de

\section{Revue d'éditorial}

Donald Brunet KINGSTON, ON

Lionel Carmant MONTREAL, QC

Colin Chalk MONTREAL, QC

K. Ming Chan EDMONTON, $A B$

Robert Chen TORONTO, ON

Mary Connolly VANCOUVER, BC

Joseph Dooley HALIFAX, NS

Daryl Fourney SASKATOON, SK

Hannah Glass SAN FRANCISCO, CA, USA

Alan Goodridge ST. JOHN's, NL

Ian Grant HALIFAX, NS

Alan Guberman OTTAWA, ON

John Hurlbert CALGARY, AB

Manouchehr Javidan VANCOUVER, BC

Patrick McDonald wINNIPEG, MB

Martin McKeown VANCOUVER, BC

Joseph Megyesi LONDON, ON

Vivek Mehta EDMONTON, AB

Steven Miller VANCOUVER, BC

Neelan Pillay CALGARY, AB

Christopher Power EDMONTON, AB

Alex Rajput SASKATOON, SK

Jean Raymond MONTREAL, QC

Gary Redekop VANCOUVER, BC

Mark Sadler HALIFAX, NS

Harvey Sarnat CALGARY, AB

John Stewart VANCOUVER, BC

Jeanne Teitelbaum MONTREAL, QC

Eve Tsai OTTAWA, ON

Shannon Venance LONDON, ON

Matt Wheatley EDMONTON, $\mathrm{AB}$

Jerome Yager EDMONTON, AB

\section{Journal Staff - Calgary, AB}

Dan Morin, Chief Executive Officer

Maggie McCallion, Designer/

Production Coordinator

Cindy Leschyshyn, Editorial Coordinator

Advertising representative/Représentant de publicité:

Brett Windle

Corporate Development Coordinator

Tel (403) 229-9575 Fax (403) 229-1661

E-mail: brett-windle@cnsfederation.org

Printer/Imprimeur:

Unicom Graphics, 4501 Manitoba Road SE Calgary, Alberta T2G 4B9
The official journal of: / La Revue officielle de:

The Canadian Neurological Society
La Société Canadienne de Neurologie

The Canadian Neurosurgical Society La Société Canadienne de Neurochirurgie

The Canadian Society of Clinical Neurophysiologists La Société Canadienne de Neurophysiologie Clinique

The Canadian Association of Child Neurology L'Association Canadienne de Neurologie Pédiatrique

The permanent secretariat for the four societies and the Canadian Neurological Sciences Federation is at:

Le secrétariat des quatre associations et du Fédération des sciences neurologiques du Canada est situe en permanence à:

7015 Macleod Trail SW, Suite 709

Calgary, Alberta, Canada T2H 2K6

The Canadian Journal of Neurological Sciences is published bimonthly. The annual subscription rate for Individuals are: $C \$ 120$ (Canada), $\mathrm{C} \$ 140$ (Foreign including USA). Subscription rates for Institutions are: $\mathrm{C} \$ 150$ (Canada), C\$170 (Foreign including USA). See www.cjns.org for details. Single copies C\$30 each plus postage and handling. Communications should be sent to: Canadian Journal of Neurological Sciences, 709 - 7015 Macleod Trail SW, Calgary, AB Canada T2H 2K6. Telephone (403) 229-9575; Fax (403) 2291661.E-mail: journal@cjns.org; Web: www.cjns.org COPYRIGHTO 2010 by THE CANADIAN JOURNAL OF NEUROLOGICAL SCIENCES INC. All rights reserved. No part of this journal may be reproduced in any form without the prior permission of The Canadian Journal of Neurological Sciences. Postage paid at Calgary, Alberta. This journal is indexed by $A b H y g$, AgBio, BIOBASE, BiolAb, BIOSIS Prev, CABS, ChemAb, CSA, CurAb, CurCont, EBSCO, Elsevier, EMBASE, ExcerpMed, HelmAb, Inpharma, JW-N, LTB, MEDLINE, MetaPress, MycolAb, NRN, NSCI, PE\&ON, PN\&I, ProtozoAb, PsycInfo, Reac, RefZh, RM\&VM, RurDevAb, SCI, SCOPUS, Swets, TDB, TOCprem.

Le Journal Canadien des Sciences Neurologiques est publié 6 fois par an. L'abonnement annuel est de 120 \$C (non-membres au Canada); 140 \$C (Etats Unis et ailleurs); l'abonnement annuel for pour les institutions est de 150 \$C (non-membres au Canada); 170 \$C (Etats Unis et ailleurs); Voir www.cjns.org pour détails. Copie simple: $30 \$ \mathrm{C}$ plus affranchissement et manutention. Toutes les communications doivent être adressés à Journal Canadien des Sciences Neurologiques, 709 - 7015 Macleod Trail SW, Calgary, AB Canada T2H 2K6. Téléphone (403) 229-9575; Fax (403) 2291661.E-mail journal@cjns.org; Web:www.cjns.org. DROITS D'AUTEUR (C) 2010: THE CANADIAN JOURNAL OF NEUROLOGICAL SCIENCES INC. Tous droits réservés. Aucune partie de ce Journal ne peut être reproduite, sous quelque forme que ce soit, sans la l'authorisation du Journal Canadien des Sciences Neurologiques. Port payé à Calgary, Alberta. Le Journal est cité et indexé dans $\mathrm{AbHyg}, \mathrm{AgBio}, \mathrm{BIOBASE}$, BiolAb, BIOSIS Prev, CABS, ChemAb, CSA, CurAb, CurCont, EBSCO, Elsevier, EMBASE, ExcerpMed, HelmAb, Inpharma, JW-N, LTB, MEDLINE, MetaPress, MycolAb, NRN, NSCI, PE\&ON, PN\&I, ProtozoAb, PsycInfo, Reac, RefZh, RM\&VM, RurDevAb, SCI, SCOPUS, Swets, TDB, TOCprem.

ISSN $0317-1671$

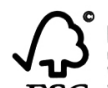

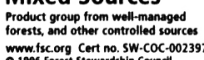




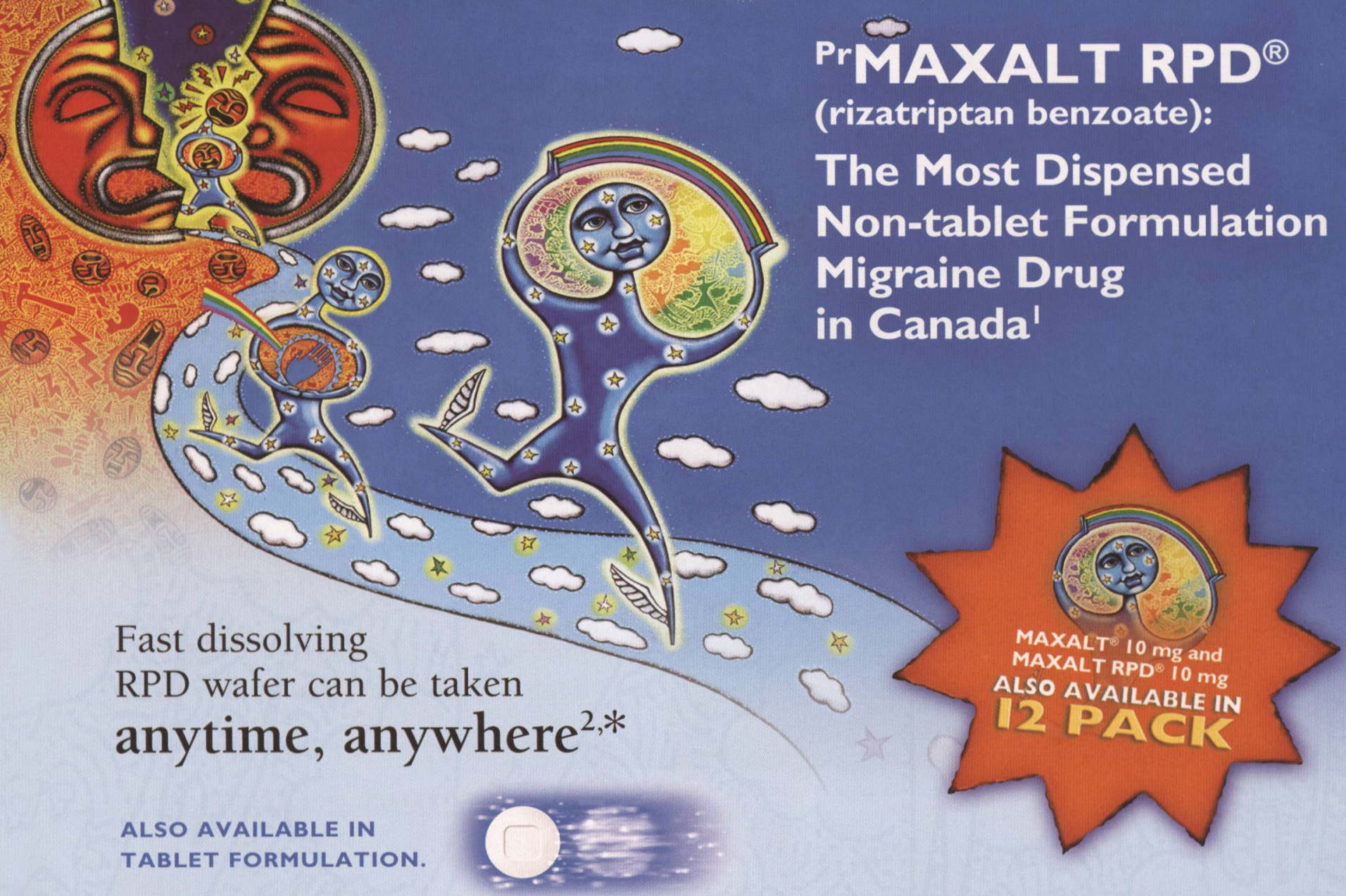

MAXALT $^{\circledast}$ (rizatriptan benzoate) is indicated for the acute treatment of migraine attacks with or without aura in adults. MAXALT ${ }^{\circledast}$ is not intended for the prophylactic therapy of migraine or for use in the management of hemiplegic, ophthalmoplegic or basilar migraine. Safety and effectiveness of MAXALT ${ }^{\otimes}$ have not been established for cluster headache, which is present in an older, predominantly male population.

MAXALT $^{\oplus}$ is contraindicated in patients with history, symptoms, or signs of ischemic cardiac, cerebrovascular or peripheral vascular syndromes, valvular heart disease or cardiac arrhythmias (especially tachycardias). In addition, patients with other significant underlying cardiovascular diseases should not receive MAXALT ${ }^{\otimes}$.

MAXALT $^{\oplus}$ is also contraindicated in patients with uncontrolled or severe hypertension.

MAXALT ${ }^{\circledast}$ is contraindicated in co-administration with monoamine oxidase (MAO) inhibitors within 2 weeks after discontinuation of treatment, and within 24 hours of administration of $5-\mathrm{HT}_{1}$ agonists or ergot-type medications. For a complete list of contraindications, please consult the Product Monograph.

The recommended single adult dose is $5 \mathrm{mg}$. The maximum recommended single dose is $10 \mathrm{mg}$.

The most common adverse events during treatment with MAXALT ${ }^{*}$ (rizatriptan benzoate) tablets $10 \mathrm{mg}$ were dizziness (8.9\%), somnolence $(8.4 \%)$, asthenia/fatigue $(6.9 \%)$, nausea $(5.7 \%)$ and pain/pressure sensation (chest, 3.1\%; neck/throat/jaw, 2.5\%; upper limb, 1.8\%).

The most common adverse events during treatment with ${ }^{P}$ MAXALT RPD ${ }^{\otimes}$ (rizatriptan benzoate) wafers $10 \mathrm{mg}$ were dizziness (8.6\%), nausea (7.0\%), dry mouth (6.0\%), somnolence (5.3\%), asthenia/fatigue (3.6\%), and pain/pressure sensation (chest, 1.7\%; neck/throat/jaw, 2.0\%; upper limb, 2.0\%).

MAXALT RPD ${ }^{\circledR}$ wafers contain phenylalanine (a component of aspartame).

*The wafer will dissolve rapidly and be swallowed with saliva. No liquid is needed to take the wafer.

$\mathrm{RPD}=$ Rapidly dissolving

References:

1. Brogan Inc. Geographic Prescription Monitor $\left(\mathrm{GPM}^{\otimes}\right)$ September 2008 to August 2009.

2. Data on file, Merck Frosst Canada Ltd.: Product Monograph, MAXALT 2009.

BEFORE PRESCRIBING MAXALT ${ }^{\circledR}$, PLEASE CONSULT THE ENCLOSED PRESCRIBING INFORMATION.

PRODUCT MONOGRAPH AVAILABLE FOR DOWNLOAD AT www.merckfrosst.com

${ }^{\circledR}$ Registered Trademark of Merck Sharp \& Dohme Corp., a subsidiary of Merck \& Co., Inc. Used under license.

Please visit our website at: www.merckfrosst.com

Merck Frosst Canada Ltd., Kirkland, Quebec
PAB

MXT-08-CDN-34381016-JA

A-5

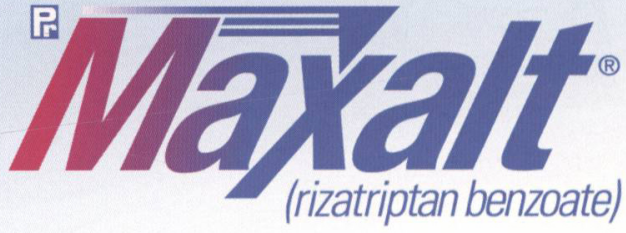

See prescribing summary on page A-14 to A-17 


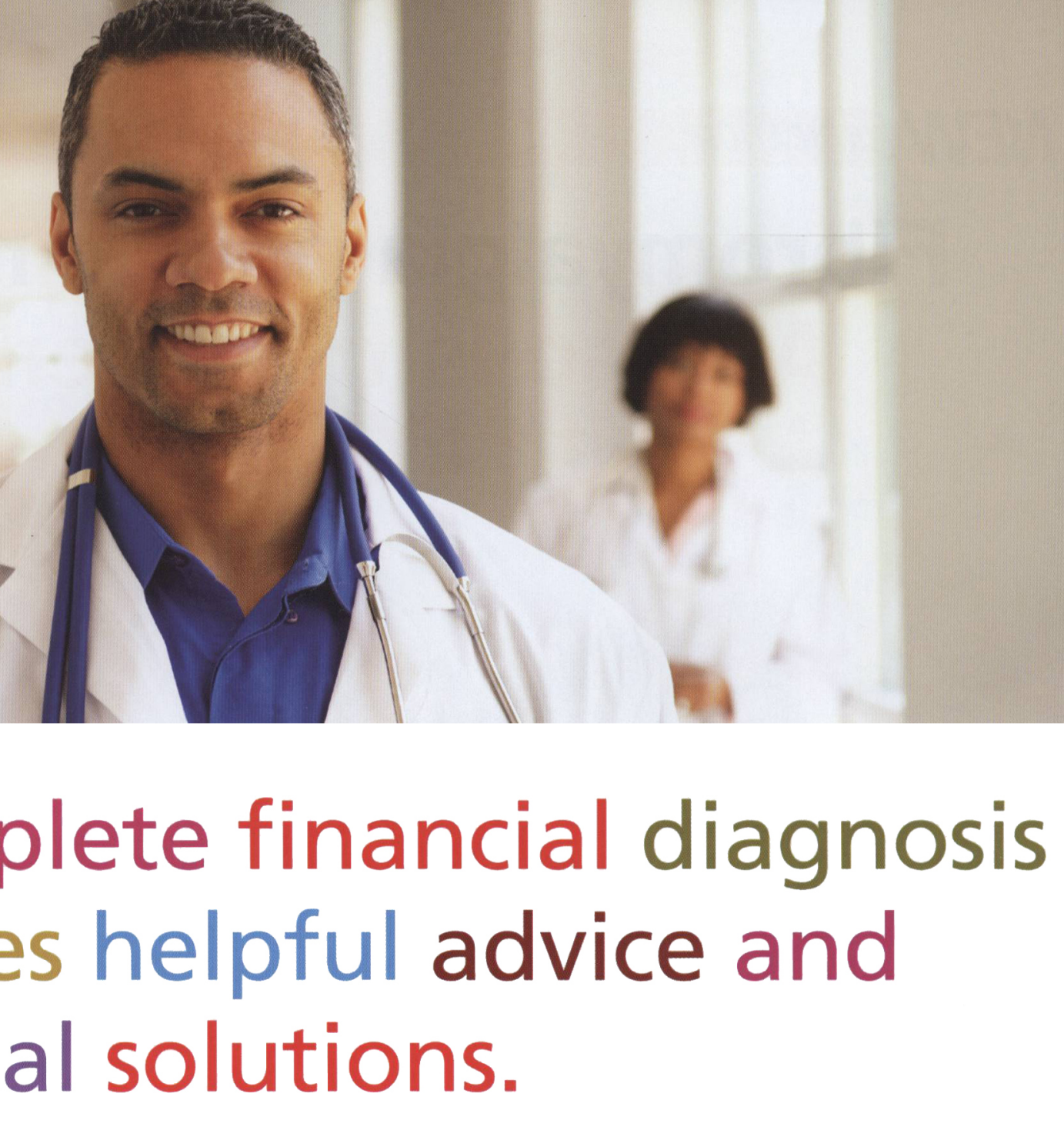

At Scotiabank, we have experts that can help you grow your business. Our Scotia Professional ${ }^{\circledast}$ Plan is a customized financial package that includes everything you need to set up and run a successful practice. You'll get competitive financing rates, flexible payback plans, and a dedicated advisor - all in one convenient package to meet your day to day banking, financing, and investment needs. It makes managing your money easy, so you can focus on serving your patients and growing your practice.

Learn more at any Scotiabank branch or visit www.scotiabank.com/professional 
